Received: 28 February 2017

Accepted: 5 June 2017

Published online: 17 July 2017

\section{Loss of type 9 adenylyl cyclase triggers reduced phosphorylation of Hsp20 and diastolic dysfunction}

Yong Li ${ }^{1}$, Tanya A. Baldwin ${ }^{1}$, Yan Wang ${ }^{1}$, Janani Subramaniam ${ }^{1}$, Anibal Garza Carbajal ${ }^{1}$, Cameron S. Brand ${ }^{1,2}$, Shane R. Cunha ${ }^{1} \&$ Carmen W. Dessauer $\mathbb{D}^{1}$

Adenylyl cyclase type 9 (AC9) is found tightly associated with the scaffolding protein Yotiao and the $I_{K s}$ ion channel in heart. But apart from potential $\mathrm{I}_{\mathrm{Ks}}$ regulation, physiological roles for $\mathrm{AC9}$ are unknown. We show that loss of AC9 in mice reduces less than $3 \%$ of total AC activity in heart but eliminates Yotiao-associated AC activity. $\mathrm{ACg}^{-1-}$ mice exhibit no structural abnormalities but show a significant bradycardia, consistent with AC9 expression in sinoatrial node. Global changes in PKA phosphorylation patterns are not altered in $\mathrm{AC}^{-1-}$ heart, however, basal phosphorylation of heat shock protein 20 (Hsp20) is significantly decreased. Hsp20 binds AC9 in a Yotiao-independent manner and deletion of AC9 decreases Hsp20-associated AC activity in heart. In addition, expression of catalytically inactive AC9 in neonatal cardiomyocytes decreases isoproterenol-stimulated Hsp20 phosphorylation, consistent with an AC9-Hsp20 complex. Phosphorylation of Hsp20 occurs largely in ventricles and is vital for the cardioprotective effects of Hsp20. Decreased Hsp20 phosphorylation suggests a potential baseline ventricular defect for $\mathrm{AC9}^{-l-}$. Doppler echocardiography of $\mathrm{AC9}^{-1-}$ displays a decrease in the early ventricular filling velocity and ventricular filling ratio $(E / A)$, indicative of grade 1 diastolic dysfunction and emphasizing the importance of local cAMP production in the context of macromolecular complexes.

The second messenger cAMP and its effector proteins regulate numerous physiological processes in heart, including pacemaker activity, stress responses and cardiac contractility ${ }^{1}$. The majority of cAMP synthesis in heart, particularly in cardiac myocytes, is ascribed to two major adenylyl cyclase isoforms, AC5 and AC6. In mice, AC5 is important for parasympathetic regulation of cAMP production and cardiac stress responses, while AC6 appears to regulate aspects of calcium handling and cardiac contractility ${ }^{1}$. Deletion of both AC5/6 suggests these two enzymes control all beta-adrenergic enhancements of L-type calcium currents ${ }^{2}$. However, physiological roles for additional AC isoforms expressed at lower levels in heart are unknown.

We previously showed that AC9, an understudied largely forskolin-insensitive AC isoform, is expressed in adult mouse cardiomyocytes and forms complexes in heart with Yotiao, an A-kinase anchoring protein (AKAP) 3 , 4 AKAPs are important scaffolds that direct the localization, regulation, and integration of cAMP-dependent PKA signaling with downstream targets. Dysregulation of AKAP organized complexes can lead to cardiac remodeling and development of heart failure ${ }^{5,6}$. For example, mAKAP (AKAP6) scaffolds AC5 to regulate cardiac stress responses while AKAP79 (AKAP5) scaffolds AC5/6 and L-type calcium channels ${ }^{5,7}$. Association of AC with AKAP complexes serves to sensitize bound PKA substrates to the effects of cAMP, by up to two orders of magnitude ${ }^{4,8}$.

In heart, AC9 is the only AC isoform to associate with Yotiao and the Yotiao- $\mathrm{I}_{\mathrm{Ks}}$ channel complex ${ }^{4}$. The $\mathrm{I}_{\mathrm{Ks}}$ channel results from the co-assembly of two subunits KCNQ1 and KCNE1. PKA phosphorylation of the anchored KCNQ1 channel subunit increases $\mathrm{I}_{\mathrm{Ks}}$ current and shortens the action potential duration to allow sufficient diastolic intervals upon increased heart rate. Mutations in either KCNQ1 or Yotiao that disrupt their interaction give rise to Long-QT syndrome (LQT1, LQT11; a potentially lethal heritable arrhythmia syndrome) ${ }^{9}$. AC9 association with Yotiao-KCNQ1 facilitates KCNQ1 phosphorylation by $\mathrm{PKA}^{4}$. In humans, we suggest that AC9 is important

${ }^{1}$ Dept. Integrative Biology and Pharmacology, McGovern Medical School, University of Texas Health Science Center, Houston, TX, 77030, USA. ²Present address: Department of Pharmacology, School of Medicine, University of California San Diego, La Jolla, CA, 92093, USA. Yong Li and Tanya A. Baldwin contributed equally to this work. Correspondence and requests for materials should be addressed to C.W.D. (email: Carmen.W.Dessauer@uth.tmc.edu) 

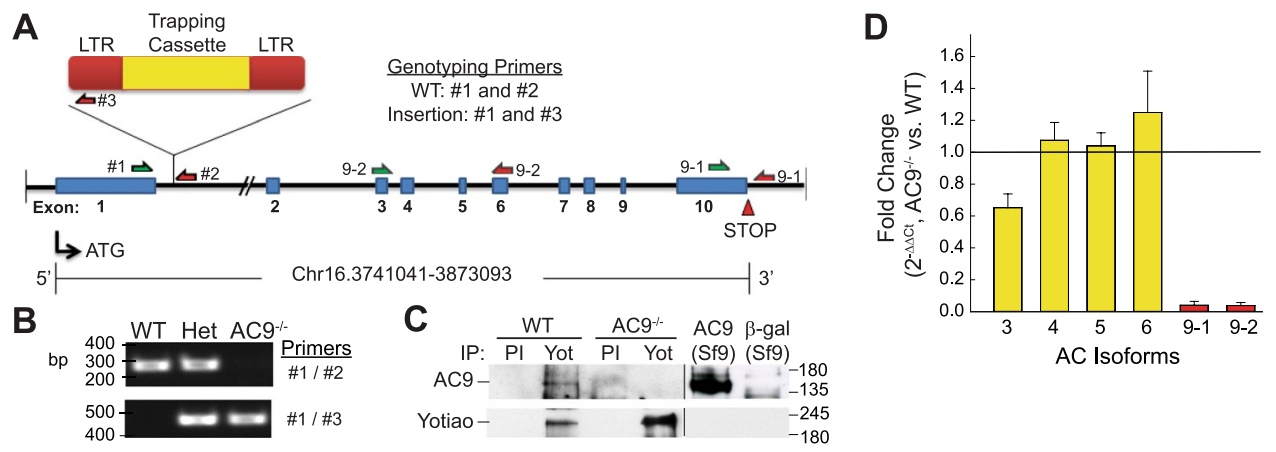

Figure 1. Design and verification of the AC9 ${ }^{-1-}$ mouse model. (A) Schematic of AC9 gene-trap strategy and genotyping primers. Intron distances are not drawn to scale. (B) PCR analysis of genotyping: lanes 1, 2, and 3 represent wild-type (WT), $\mathrm{AC} 9^{+--}$(Het) and $\mathrm{AC}^{-1-}$, respectively. (C) AC9 protein levels were detected by immunoprecipitation of pre-immune (PI) or Yotiao complexes from WT and AC9 KO heart extracts followed by western blotting (WB) with anti-AC9 antibody. AC9 protein is not detectable in total heart extracts by WB $(n=5)$. (D) Real time PCR of cardiac AC isoforms in AC9 ${ }^{-1-}$ heart, normalized to WT expression levels $(n=3$; mice 1 month of age). Loss of AC9 mRNA was confirmed with primer sets 9-1 and 9-2. Full-length WBs are presented in Supplementary Fig. S6.

for repolarization of heart. However since a functional $\mathrm{I}_{\mathrm{Ks}}$ is largely absent in adult mice, additional potential roles for AC9 in heart are unknown.

In this report we show that AC9 accounts for less than 3\% of total AC activity in mouse heart, yet represents all the Yotiao-associated AC activity. Loss of AC9 expression does not alter cardiac structure nor global PKA phosphorylation, but results in decreased PKA phosphorylation of heat shock protein 20 (Hsp20). PKA phosphorylated Hsp20 has previously been shown to be cardioprotective ${ }^{10-12}$. Molecular analyses indicate that Hsp20 is associated with AC9 in a Yotiao-independent manner. Loss of AC9 decreases Hsp20-associated AC activity in heart, while overexpression of catalytically inactive AC9 in neonatal cardiomyocytes decreases isoproterenol-stimulated Hsp20 phosphorylation. Finally, AC9 deletion gives rise to a grade 1a left ventricular diastolic dysfunction with preserved ejection fraction, consistent with a cardioprotective role for AC9.

\section{Results}

Genetic ablation of AC9 results in preweaning subviability. AC9 is ubiquitously expressed but physiological roles for AC9 have been largely ignored. To investigate the in vivo function of AC9, we utilized a genetrap strain of AC9 obtained from the Mutant Mouse Regional Resource Center, a NIH strain repository. The AC $9^{-1-}$ strain was created by Lexicon, Inc. using a retroviral insertion between exon 1 and 2 (Fig. 1A). The mouse genotypes were determined by PCR assay (Fig. 1B). AC9 protein is not detectable by western blotting in heart tissue homogenates and available antibodies against AC9 do not work well for immunoprecipitation. Therefore to confirm the lack of AC9 protein expression, we probed the Yotiao-AC9 complex which is tightly associated in mouse and guinea pig heart ${ }^{4}$. AC9 protein is detectable in immunoprecipitates of Yotiao from wild type (WT) hearts but not $\mathrm{AC}^{-/-}$(Fig. 1C). Multiple isoforms of AC are expressed in mouse adult cardiomyocytes, including $\mathrm{AC} 3,4,5,6$, and $9^{4}$; quantitative PCR of these $\mathrm{AC}$ isoforms from WT and $\mathrm{AC} 9^{-1-}$ shows a $35+1-9 \%$ decrease in AC3 mRNA and complete loss of AC9, but no significant difference in other AC isoforms (Fig. 1D). AC3 protein was undetectable in lysates by western blotting.

Unpublished observations of FA Antoni suggested that conventional targeted deletion of AC9 results in early embryonic lethality in mice ${ }^{13}$. Although viable, we noted abnormal genotype frequencies for heterozygous mating pairs after backcrossing to C57BL/6 J (18\% WT, 75\% Het, 7\% KO; $\mathrm{n}=68, \mathrm{P}=0.002)$. The preweaning subviable homozygous phenotype with incomplete penetrance is also reported for another Adcy $9^{-1-}$ strain (Adcy9tm1b(EUCOMM)Wtsi) created as part of the International Knockout Mouse Consortium ${ }^{14}$.

Deletion of AC9 results in loss of Yotiao-associated AC but not significant changes in total AC activity. AC9 mRNA and/or protein has previously been detected in both cardiac fibroblasts and myocytes ${ }^{4,15}$, however the degree to which AC9 contributes to total AC activity is unknown. Initial measurements of basal and Gos-stimulated AC activity showed no difference between cardiac membranes isolated from WT versus AC9 ${ }^{-1-}$ mice (Fig. 2A). To potentially unmask AC activity stemming from AC9, we used a P-site inhibitor that displays $>100$ fold selectivity for AC5/6 over AC9 (Fig. 2B) ${ }^{16}$. No significant difference in total AC activity is observed, even at SQ 22,536 concentrations that inhibit $70-90 \%$ of AC5/6, but only $20-30 \%$ of AC $9(30-100 \mu M)$. From this data, we estimate that AC9 represents less than 3\% of total heart AC activity. In order to detect AC9 activity, we examined its association with specific AKAP complexes ${ }^{3,4}$. Heart extracts subjected to immunoprecipitation of Yotiao show significant AC activity that is pulled down with Yotiao in WT but not $\mathrm{AC}^{-1-}$ (Fig. 2C). This is consistent with our previous findings that AC9 is the only AC isoform associated with Yotiao in heart ${ }^{4}$. Although AC9 can also bind AKAP79/150 ${ }^{17}$, it does not significantly contribute to the AC activity associated with AKAP79/150 in heart (Fig. 2D). 
A

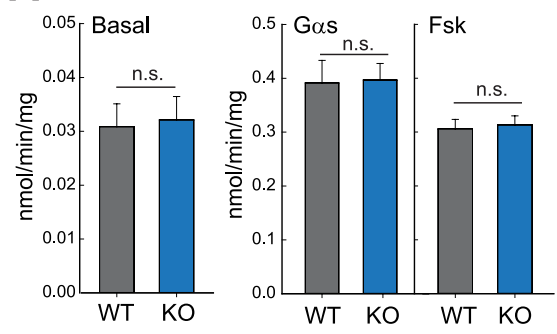

B

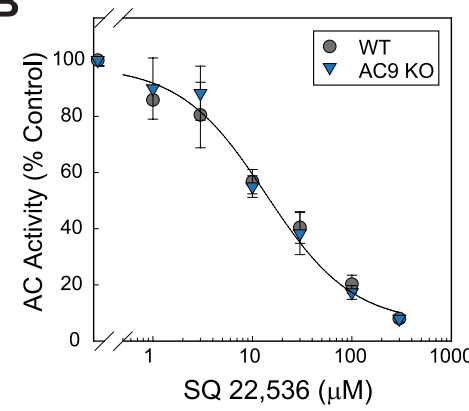

C

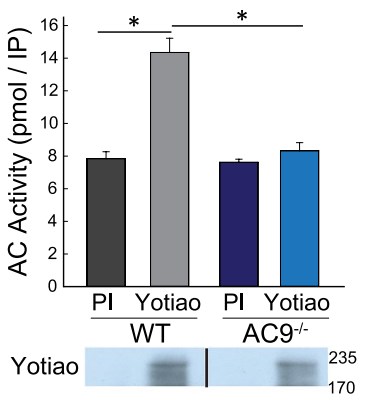

D

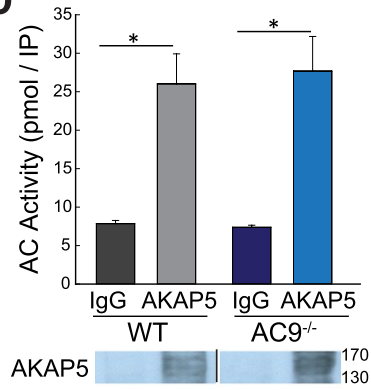

E

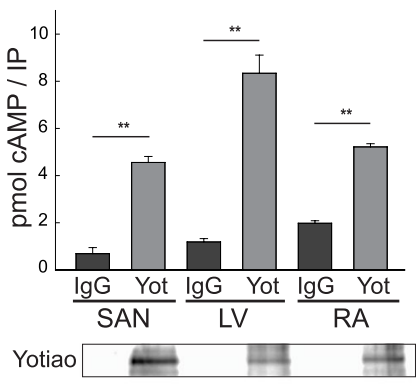

$\mathbf{F}$

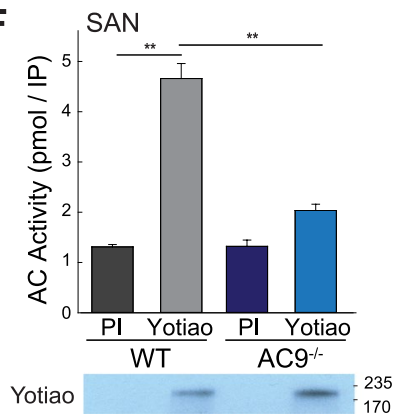

Figure 2. AC9 activity and AKAP association in heart. (A,B) Membranes were prepared from 6 wk WT versus AC $9^{-1-}$ heart. (A) AC activity was measured under basal conditions and upon stimulation with $300 \mathrm{nM}$ Gos or $50 \mu \mathrm{M}$ forskolin ( $\mathrm{n}=4$, performed in duplicate or triplicate). (B) AC activity was measured in the presence of increasing concentrations of SQ22,536 in the presence of $300 \mathrm{nM} \mathrm{G \alpha s}(\mathrm{n}=3$, performed in duplicate). (C,D) Heart extracts from WT or AC9 ${ }^{-1-}$ mice were subjected to immunoprecipitation (IP) with pre-immune (control) or anti-Yotiao (C) and control IgG or anti-AKAP5 (D). AKAP-associated AC activity was stimulated with $300 \mathrm{nM}$ Gos and measured $(\mathrm{n}=3-4)$. Data are shown as mean $+/-$ SD. A portion of the IP's from $(\mathbf{C}$ and D) were subjected to WB analysis for the appropriate AKAP. (E) Yotiao-associated AC activity was measured from WT sinoatrial node (SAN), left ventricle (LV), and right atrium (RA) tissue homogenates as in panel (C). Immunoblot of Yotiao from IPs is shown below. (F) Yotiao-associated activity from WT and AC9 ${ }^{-1-}$ SAN as measured in panel $(C)$; Yotiao immunoblot is shown below $(n=3)$. Full-length WBs are presented in Supplementary Fig. S6.

\begin{tabular}{|l|l|l|l|l|}
\hline \multirow{2}{*}{ Sex } & \multirow{2}{*}{ Age $(\mathbf{m o})$} & \multicolumn{2}{|l|}{ Average Heart Rate $(\mathbf{b p m})$} & \\
\cline { 3 - 4 } & WT & AC9 $^{-1-}$ & p-value \\
\hline Male/Female & $1-2$ & $433+/-5(\mathrm{n}=13 / 6)$ & $409+/-7(\mathrm{n}=12 / 6)$ & 0.008 \\
\hline Male & $5-7$ & $445+/-6(\mathrm{n}=10)$ & $400+/-7(\mathrm{n}=11)$ & 0.0002 \\
\hline
\end{tabular}

Table 1. Deletion of AC9 gives rise to bradycardia.

Reduced heart rate in the absence of AC9. Functional analysis of WT and $\mathrm{AC} 9^{-1-}$ mice revealed a significant reduction of heart rate under isoflurane in both male and female mice for the two age groups examined (Table 1$)$. Body weight is unaltered in male $(21.1+/-0.2$ versus $21.6+/-0.3 \mathrm{~g}, 6$ months $)$ and female mice (15.3 $+/-0.2$ versus $15.6+/-0.2 \mathrm{~g}, 4$ months). No structural abnormalities were noted and myocardial performance index $(0.9+/-0.1$ versus $0.9+/-0.1)$, ejection fraction $(53+/-3$ versus $51+/-4)$ and percent fractional shortening $(27+/-2$ versus $26+/-3)$ were all unchanged, as assessed by M mode imaging of mice 3-7 months (Supplemental Table 1). Alterations in heart rate suggest a role for AC9 in sinoatrial node (SAN). Yotiao-AC9 complex is detected throughout the heart, including the left ventricle, atria, and the SAN, as measured by AC9 activity associated with Yotiao immunoprecipitation (Fig. 2E). As shown for total heart, deletion of AC9 abolishes Yotiao-associated activity in SAN (Fig. 2F), consistent with a role for AC9 in heart rate control. Correct dissection of SAN is confirmed by the presence of connexin 45 but not connexin 43 (Supplemental Fig. S1) ${ }^{18}$.

Global PKA phosphorylation is unaltered but Hsp20 phosphorylation is decreased in $\mathrm{AC}^{-1-}$. In order to determine if AC9 deletion alters CAMP signaling, we used intraperitoneal injection of saline or isoproterenol in WT and $\mathrm{AC}^{-/-}$mice to evaluate changes in phosphorylation of PKA targets. Global changes in PKA phosphorylation at baseline or after isoproterenol injection are not detected (Fig. 3A), suggesting that AC9 does not significantly alter sympathetic responses. However, we detect a large decrease in the basal phosphorylation state of Hsp20 in the absence of beta-adrenergic stimulation (Fig. 3B). Hsp20 is a known PKA $\operatorname{target}^{19,20}$ and inhibition of PKA activity by H89 blocks isoproterenol-stimulated phosphorylation of Hsp20 in rat 
A
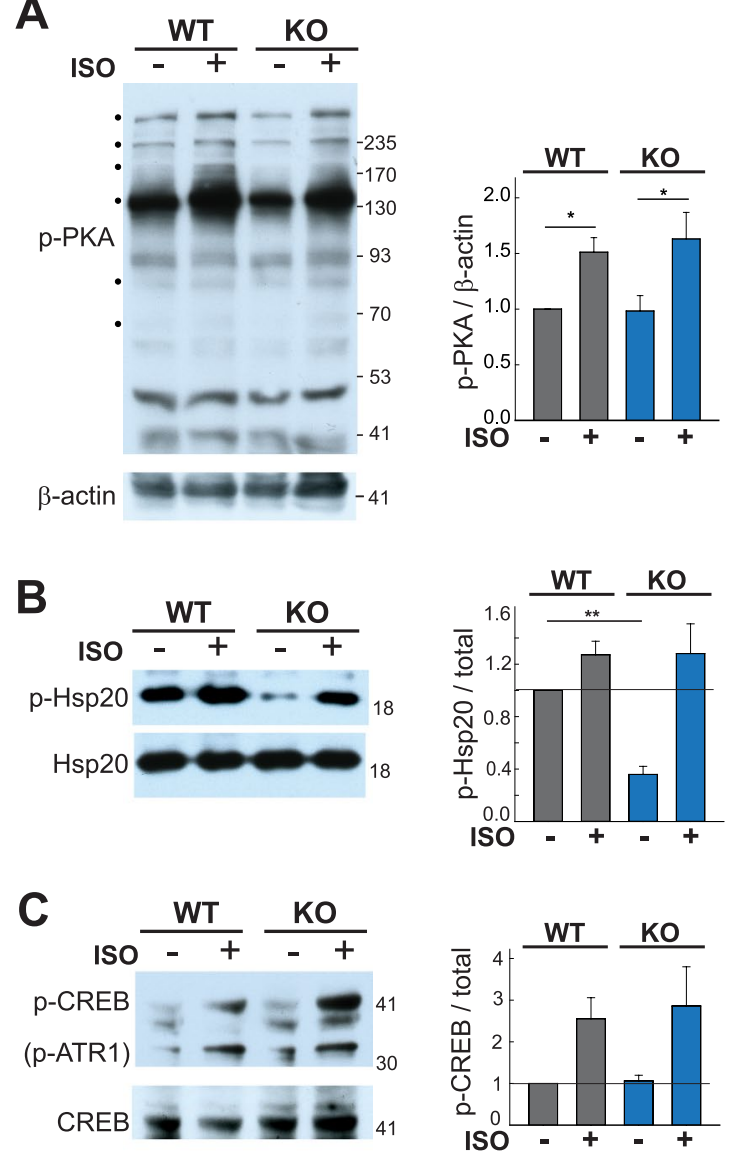

D
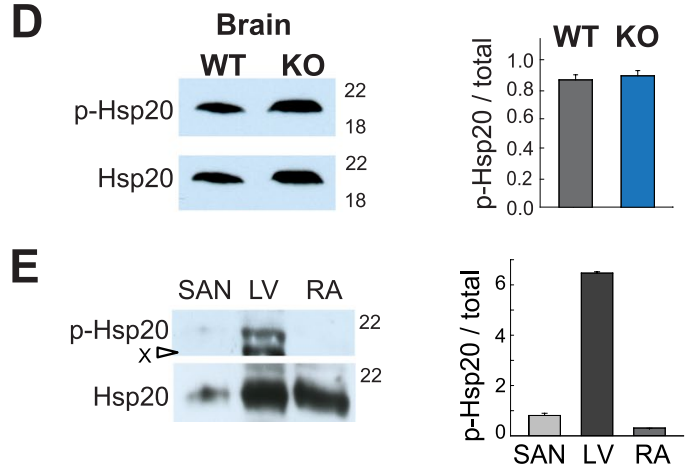

Figure 3. Decreased PKA phosphorylation of $\mathrm{Hsp} 20$ in $\mathrm{AC}^{-1-}$. WT and $\mathrm{AC}^{-1-}$ mice were injected with saline or isoproterenol ( $2 \mu \mathrm{g} / \mathrm{g}$ body weight, IP). Animals were sacrificed $4 \mathrm{~min}$ later and heart tissue was harvested. Cardiac extracts were prepared in the presence of phosphatase inhibitors. Equal protein supernatants were subjected to WB analysis with (A) anti-p-PKA substrate, (B) anti-p-Hsp20, and C) anti-p-CREB. Quantitation of phospho-PKA was normalized to beta-actin levels (A) while the corresponding total protein was quantitated by WB $(n=5-7)$ and the ratio of phosphoprotein to total was quantitated for p-Hsp20 (B) and p-CREB (C). (D,E) WB and quantitation of the ratio of phosphorylated to total Hsp20 in D) brain from WT and $\mathrm{AC} 9^{-1-}$ mice $(\mathrm{n}=3)$ and $(\mathrm{E})$ sinoatrial node, left ventricle, and right atrium $(\mathrm{n}=3)$. Graphs for quantitation of the ratio of phosphorylated to non-phosphorylated protein are shown to the right for each panel. $* * \mathrm{P}<0.01$ t-test on raw intensity values. Full-length WBs are presented in Supplementary Fig. S6.

neonatal cardiomyocytes (Supplemental Fig. S2). Phosphorylation of other PKA targets such as troponin I, CREB, and phospholamban (PLN) are unaltered in the knockout (Fig. 3C, Supplemental Fig. S3). No change in basal Hsp20 phosphorylation was observed in $\mathrm{WT}$ and $\mathrm{AC}^{-1-}$ brain lysates (Fig. 3D), suggesting that AC9 regulates Hsp20 in a tissue specific manner. Hsp20 total protein is present at very low levels in SAN node (only $11+/-9 \%$ of that in LV; Fig. 3E), while basal phosphorylation of Hsp20 is only significantly detected in left ventricle and not atrium (Fig. 3E).

To determine if AC9 is present in Hsp20-containing complexes, we measured the AC activity present in Hsp20 immunoprecipitates in WT and $\mathrm{AC}^{-1-}$ heart extracts. Not only is a significant amount of AC activity complexed 

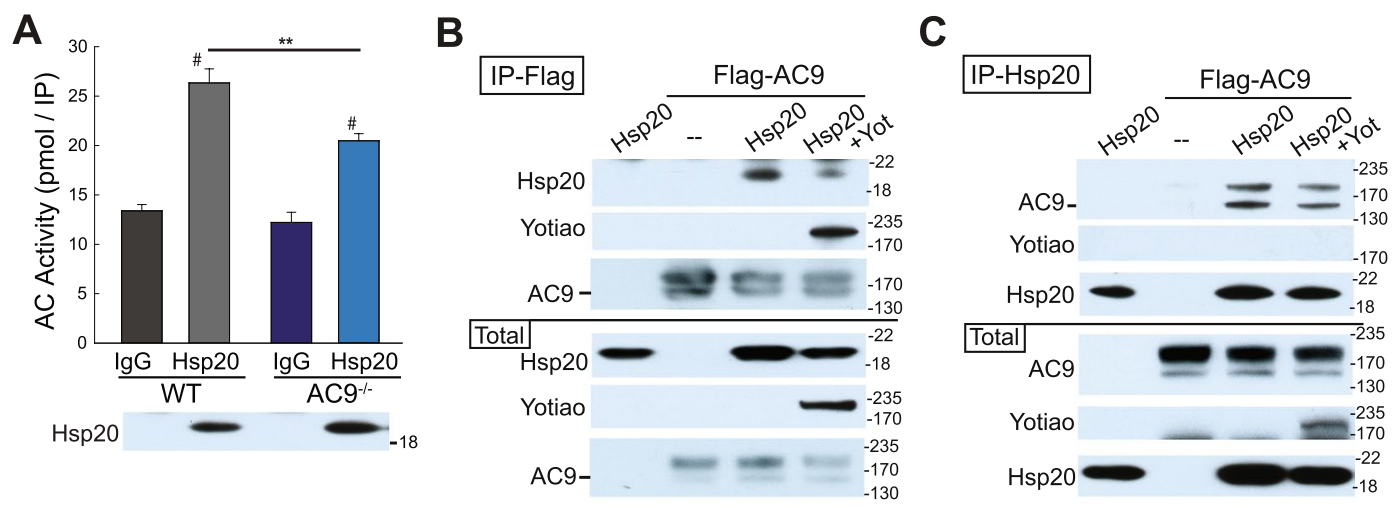

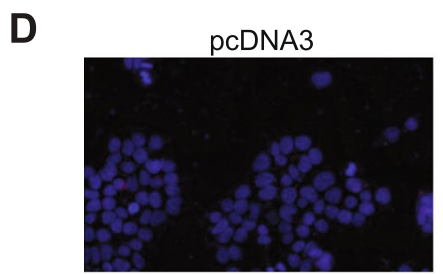

YFP-AC9 + Hsp20

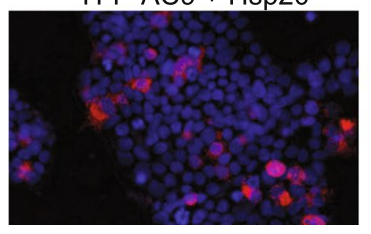

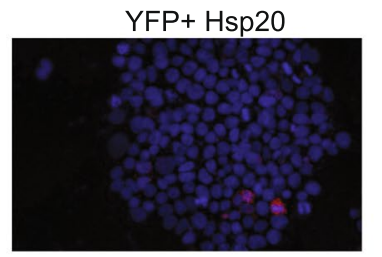

YFP-AC9 + G $\beta \gamma$

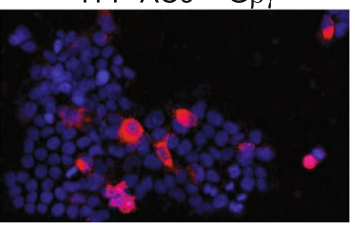

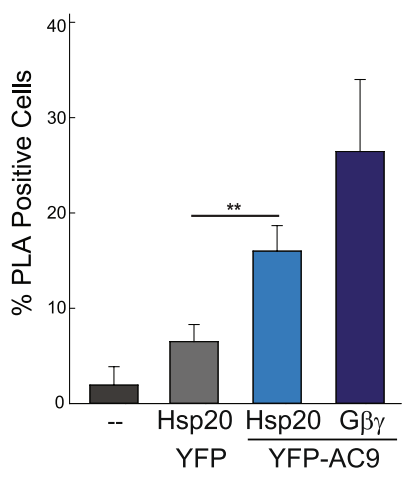

Figure 4. AC9 association with Hsp20 is Yotiao independent. (A) Heart extracts from WT or AC9-/- mice were subjected to IP with IgG (control) or anti-Hsp20. Hsp20-associated AC activity was stimulated with $300 \mathrm{nM}$ Gos $\left(\mathrm{n}=4{ }^{*} \mathrm{P}<0.001\right.$ IgG versus Hsp20; $* * \mathrm{P}<0.01$ WT versus $\left.\mathrm{AC}^{-1-}\right)$. (B,C) HEK293 cells were transfected with Flag-tagged AC9, myc-tagged Yotiao, and V5-tagged Hsp20 as indicated. Cell lysates were immunoprecipitated with anti-Flag (B) or anti-Hsp20 (C). AC9-Hsp20 protein complexes were detected by WB $(\mathrm{n}=3)$. (D) Proximity ligation assay for Hsp20 and YFP-tagged AC9. HEK293 cells were transfected with the indicated plasmids. Representative images are shown. PLA signals were quantified by high content microscopy (positive cells defined as 4 signals or "dots" per cell; 2000 cells imaged per condition). $* \mathrm{P}<0.05 \mathrm{t}$-test, $\mathrm{n}=3$. Full-length WBs are presented in Supplementary Fig. S6.

with Hsp20 in WT heart, this activity is decreased by $35+/-6 \%$ in $\mathrm{AC}^{-/-}$(Fig. 4A). Additional AC isoforms may associate with Hsp20 to facilitate isoproterenol-stimulated PKA phosphorylation. Interaction of AC9 and Hsp20 appears independent of Yotiao, as no detectable Hsp20 is associated with Yotiao in heart (Supplemental Fig. S4). AC9 and Hsp20 interactions can also be observed in HEK293 cells. A complex of AC9-Hsp20 is detected upon immunoprecipitation of either Flag-tagged AC9 or Hsp20 (Fig. 4B,C). Note, we consistently observe a 72 $+/-7 \%$ reduction of AC9-Hsp20 binding in the presence of Yotiao, suggesting that Yotiao may compete with Hsp20 for AC9 interactions. Finally, AC9-Hsp20 interactions are also detected by proximity ligation assay (PLA) in HEK293 cells (Fig. 4D). The high selectivity of PLA relies on double recognition of a protein complex by two oligonucleotide-conjugated secondary antibodies. Oligonucleotides in close proximity allows for rolling circle amplification that can be visualized (Fig. 4D, red dots). YFP-AC9 and Hsp20 display a significant signal by PLA as compared to YFP alone. G $\beta \gamma$ binds to the N-terminus of AC9 and serves as a positive control ${ }^{21}$.

AC9 binding to an Hsp20 complex should regulate local cAMP production and subsequent Hsp20 phosphorylation. If this is the case, displacing AC9 with a catalytically inactive enzyme would lead to reduced local cAMP and Hsp20 phosphorylation. Mutation of D399 to alanine deletes a key metal-binding residue in the active site of AC9, reducing Gos-stimulated activity by $>90 \%$ (Supplemental Fig. S5). Adenoviral expression of AC9-D399A in rat neonatal cardiomyocytes significantly decreased isoproterenol-stimulation of Hsp20 (by $77+/-6 \%$ ), as compared to non-infected or GFP-infected cells (Fig. 5). This is consistent with decreased Hsp20 phosphorylation and Hsp20-associated AC activity in $\mathrm{AC}^{-1-}$ heart.

Protective role for AC9 against diastolic dysfunction. The decrease in baseline Hsp20 phosphorylation in $\mathrm{AC}^{-1-}$ suggests a potential loss of the cardioprotective effects of PKA phosphorylated Hsp20 $20^{10-12}$. Therefore, we measured overall left ventricular function using pulsed-wave Doppler echocardiography of early (E) and late (A) blood flow velocities through the mitral valve combined with tissue Doppler imaging of the mitral valve annulus (E' and A' velocity). Diastolic relaxation consists of four phases: isovolumetric relaxation, early rapid ventricular filling, slow filling or diastasis, and atrial contraction. Of these, the early filling phase (E wave) appears significantly reduced in $\mathrm{AC}^{-1-}$ mice (Table 2 and Fig. 6). This was confirmed by tissue Doppler, 

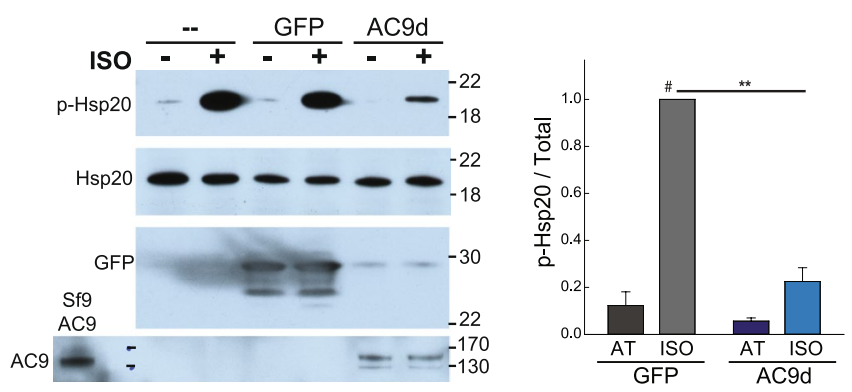

Figure 5. Expression of catalytically inactive AC9 decreases isoproterenol-stimulated phosphorylation of Hsp20 in rat neonatal cardiac myocytes (RNCMs). Cells were infected with GFP control or catalytically inactive AC9-D399A (AC9d) adenoviruses for $50 \mathrm{hr}$. RNCMs were treated with vehicle (AT) or isoproterenol $(1 \mu \mathrm{M})$ for 5 min prior to cell lysis. The ratio of $\mathrm{p}-\mathrm{Hsp} 20$ to total Hsp20 was quantitated by WB. ${ }^{*}, * * \mathrm{P}<0.01 \mathrm{t}$-test, $\mathrm{n}=4$. Full-length WBs are presented in Supplementary Fig. S6.

\begin{tabular}{|l|l|l|l|}
\hline Parameter & WT & AC9 $^{-/-}$ & P value \\
\hline Mitral flow doppler & $20+/-1$ & $15+/-1$ & 0.004 \\
\hline Peak velocity, E' (mm/s) & $16.5+/-0.6$ & $16.3+/-0.5$ & n.s. \\
\hline Peak velocity, A' (mm/s) & $51+/-2$ & $52+/-1$ & n.s. \\
\hline Aortic ejection time (AET, ms) & $24+/-2$ & $27+/-2$ & n.s. \\
\hline Isovolumic Relaxation Time (ms) & $20+/-1$ & $20+/-1$ & n.s. \\
\hline Isovolumic Contraction Time (ms) & $41+/-2$ & $43+/-2$ & n.s. \\
\hline Mitral valve A (cm/s) & $64+/-2$ & $54+/-2$ & 0.005 \\
\hline Mitral valve E (cm/s) & $0.88+/-0.05$ & $1.2+/-0.1$ & 0.04 \\
\hline A'/E' & $1.57+/-0.08$ & $1.27+/-$ & 0.07 \\
\hline MV E/A & $33+/-1$ & $38+/-3$ & n.s., 0.1 \\
\hline MV E/E' & $0.9+/-0.1$ & $0.9+/-0.1$ & n.s. \\
\hline Myocardial Performance Index (MPI) & & & \\
\hline
\end{tabular}

Table 2. Cardiac parameters for $\mathrm{WT}$ and $\mathrm{AC} 9^{-1-}$ mice. ${ }^{*}$ Mean $+/-\mathrm{SE}$ is given for male animals 3-7 months.

measuring the early diastolic mitral annular velocity (E') which is preload independent. Trends towards increased filling pressures (E/e') are observed but never reach significance.

\section{Discussion}

AC9 is the most divergent in sequence of the nine mammalian transmembrane AC isoforms. It is relatively insensitive to forskolin activation and remains the least characterized. It was originally cloned as a calcineurin-inhibitable $\mathrm{AC}$ isoform ${ }^{22}$, although it is unclear if calcineurin and other reported cellular regulators have direct or indirect mechanisms of action ${ }^{23}$. AC9 mRNA is ubiquitously expressed, particularly in the hippocampus $^{24}$, and the knockout of AC9 was thought to be embryonic lethal ${ }^{13}$. Therefore, physiological roles for AC9 have been largely ignored. However, despite a preweaning subviability, AC9 ${ }^{-1-}$ mice show no obvious size or structural abnormalities. AC9 expression is important for human neutrophil chemotaxis ${ }^{25}$, while ADCY9 gene polymorphisms are linked to asthma, mood disorders, and body weight ${ }^{26-28}$. In heart, AC9 mRNA and/or protein has been detected in both cardiomyocytes and fibroblasts, however, our estimates using P-site inhibitors suggest that AC9 represents less than 3\% of total heart AC activity. Despite the low levels of AC9, Yotiao-associated AC activity is abolished in the knockout while AKAP79-associated AC activity is unchanged. Although AKAP79 can bind to AC9 in cell culture experiments, AKAP79 scaffolds the highly expressed AC5/6 isoforms in heart ${ }^{17,29}$. The association with Yotiao is consistent with a role for AC9 in regulation of $\mathrm{I}_{\mathrm{Ks}}$ channels and potentially long QT syndrome, for which mutations of $\mathrm{I}_{\mathrm{Ks}}$ and Yotiao are well known'.

Bradycardia. LQT1 patients (KCNQ1 mutations) often display reduced heart rate, particularly with exercise $^{30,31}$. AC9 is proposed to regulate PKA phosphorylation of KCNQ1 via its scaffolding to Yotiao ${ }^{4}$, however, the interpretation of a bradycardia phenotype is complicated in mice. Unlike humans, standard $\mathrm{I}_{\mathrm{Ks}}$ currents are largely absent in adult mouse myocytes ${ }^{32-34}$. We do not detect endogenous KCNQ1 from heart lysates or by immunoprecipitation of Yotiao or KCNQ1 ${ }^{4}$. Moreover, knockout of KCNQ1 does not alter heart rate or QT interval at baseline, although these features are prolonged when challenged with nicotine ${ }^{35}$. Thus it is unclear if the bradycardia in $\mathrm{AC}^{-1-}$ is due to decreased phosphorylation of KCNQ1 or another, yet identified, Yotiao-associated $\mathrm{K}^{+}$ channel in mouse sinoatrial node.

AC9 binds Hsp20 and regulates basal Hsp20 phosphorylation. Although global PKA phosphorylation is unchanged, deletion of AC9 has a pronounced effect on the basal phosphorylation of Hsp20. 
A
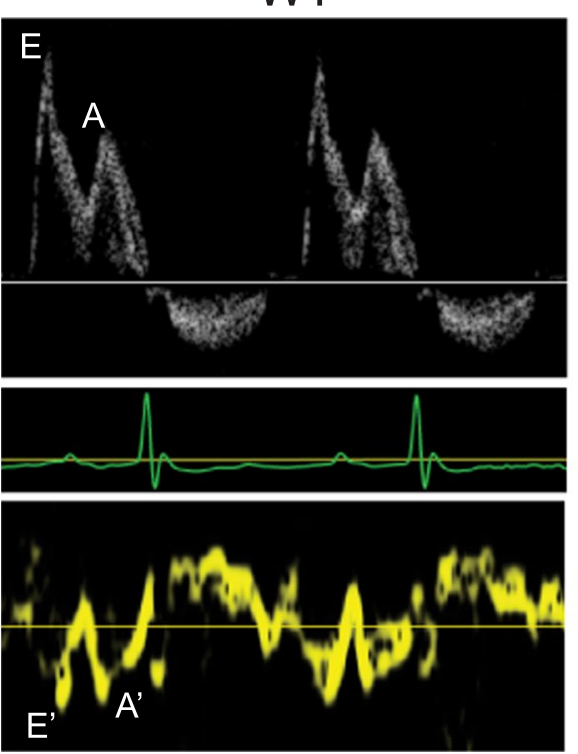

B

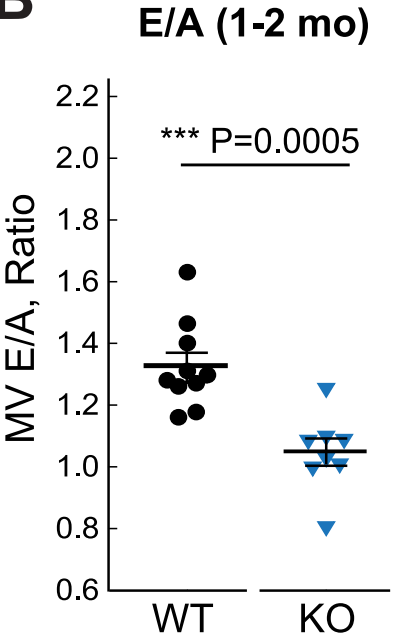

E/A (3-7 mo)

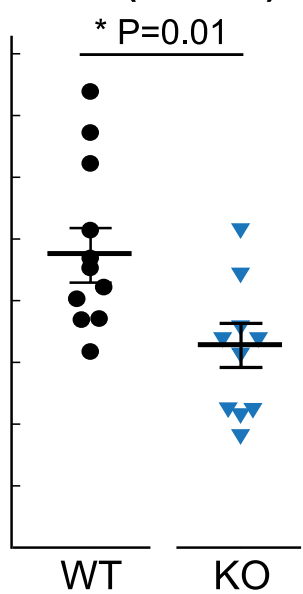

AC9-1-
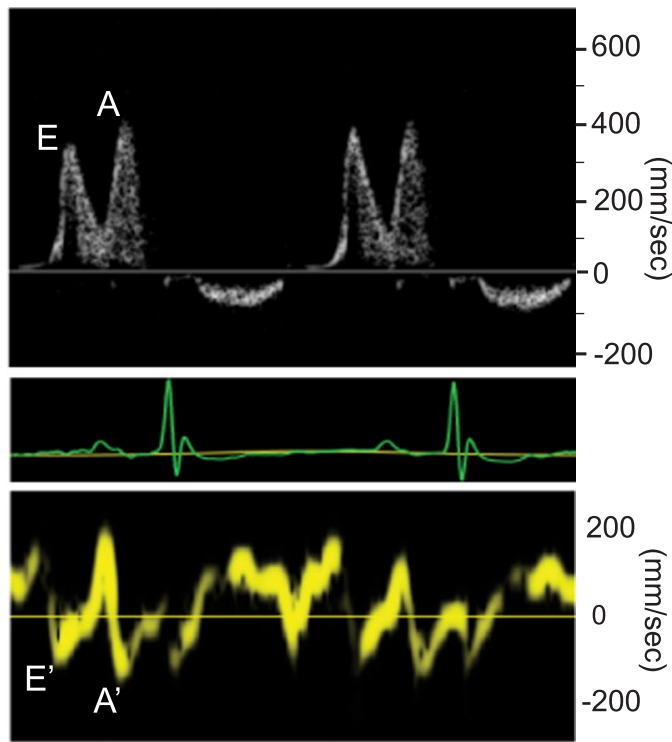

200
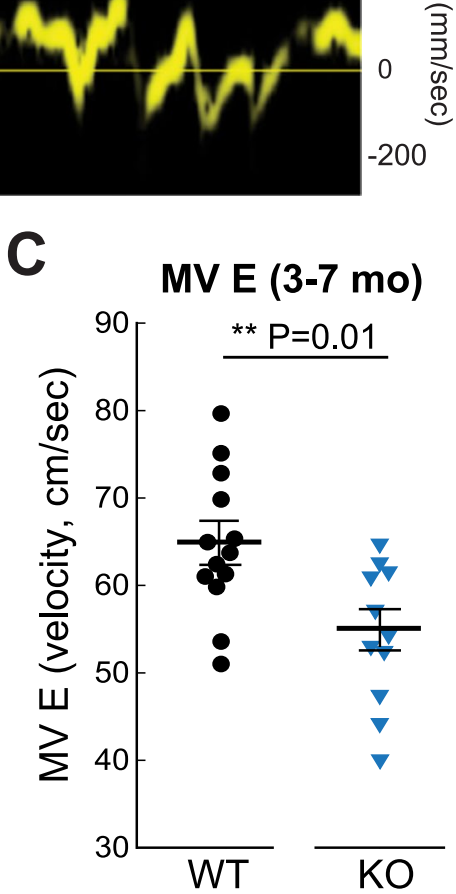

Figure 6. Cardiac parameters for WT and $\mathrm{AC}^{-1-}$ mice. (A) Pulsed-wave and tissue Doppler recordings for female WT and $\mathrm{AC} 9^{-1-}$ littermates (3 month) showing the relative amplitudes of the early ventricular filling (E wave) and the late filling caused by atrial contraction (A wave) (ECGs are shown below for each animal). (B) Quantitation of E/A ratio at $1-2\left(\mathrm{n}=10 \mathrm{WT}\right.$ and $8 \mathrm{AC} 9^{-1-}$; males, $\left.\mathrm{P}=0.0005\right)$ or $3-7$ months $(\mathrm{n}=11 \mathrm{WT}$ and $11 \mathrm{AC}^{-1-}$; males and females, $\left.\mathrm{P}=0.005\right)$. (C) Quantitation of mitral valve E wave at 3-7 months $(\mathrm{n}=13 \mathrm{WT}$ and $\left.11 \mathrm{AC}^{-1-}, \mathrm{P}=0.005\right)$. Mean $+/-\mathrm{SE}$.

Despite its name, Hsp20 (also known as HspB6) is not heat inducible but belongs to a group of ubiquitously expressed small heat shock proteins that are abundant in cardiac, smooth, and skeletal muscle ${ }^{36,37}$. Hsp20 is up-regulated in response to cellular stress and/or damage and its expression is cardioprotective against prolonged beta-agonist induced hypertrophy, ischemia/reperfusion injury, and the cardiotoxic effects of doxorubicin ${ }^{36-38}$. Phosphorylation of Ser16 on Hsp20 by PKA is increased under cardiac stress and is essential for its protective effects after ischemia/reperfusion injury ${ }^{10-12,39}$. Hsp20 is found in association with numerous complexes in heart including actin/ $\alpha$-actinin, 14-3-3, phosphorylated Akt, PP1-PLN, and members of an AKAP-Lbc complex implicated in the development of cardiac hypertrophy (reviewed in refs 36, 37 and 40). Disruption of Hsp20 association with PDE4D ${ }^{39}, \mathrm{PKD}^{11}$, or AKAP-Lbc ${ }^{42}$ abolish the protective effects of Hsp20 in the heart.

Hsp20 is associated with AC9 in heart and in HEK293 cells, as determined by IP and PLA assays. It is not clear if AC9 directly binds Hsp20 or if the association is mediated by an Hsp20 binding partner. However, we see no evidence for Hsp20 interaction with Yotiao in heart; in HEK293 cells, expression of Yotiao actually reduces AC9-Hsp20 interactions. Although AC9 clearly binds Hsp20, other AC isoforms in heart must also associate with Hsp20-containing complexes, as $65+/-4 \%$ of Hsp20-associated AC activity still remains in $\mathrm{AC}^{-1-}$ heart. Expression of a catalytically inactive mutant of AC9 in neonatal cardiomyocytes decreases 
isoproterenol-stimulated Hsp20 phosphorylation by $77+/-6 \%$. Therefore, overexpression of catalytically inactive AC9 may prevent Hsp20 from associating with endogenous AC9 and other AC isoforms in cardiomyocytes, reducing local cAMP production necessary for PKA phosphorylation of Hsp20.

Protective role for AC9 against diastolic dysfunction. Despite the fact that AC9 associates with the Yotiao- $\mathrm{I}_{\mathrm{Ks}}$ complex, it wasn't clear if deletion of AC9 would display alterations in the relaxation of heart muscle in mice, given the reported lack of $\mathrm{I}_{\mathrm{Ks}}$ currents. Surprisingly, the early filling phase (E wave) of diastolic relaxation appears significantly reduced in $\mathrm{AC}^{-1-}$ mice. This was confirmed by tissue Doppler imaging. A slower E wave is associated with a stiffer left ventricular wall, increasing the back pressure to slow blood flow velocity. This gives rise to a lower E/A ratio in $\mathrm{AC}^{-/-}$, an accepted clinical marker of diastolic dysfunction. The resulting phenotype is categorized as a grade 1a with preserved ejection fraction. Patients with LQTS (LQT1 and LQT2) also show diastolic dysfunction, with a significantly slower E' and increased left atrial volume index as compared to healthy subjects $^{43}$. However, the QT interval is not increased in $\mathrm{AC}^{-1-}$, suggesting the lower E/A ratio may not reflect altered $\mathrm{K}^{+}$channel regulation involved in repolarization, but rather the loss of Hsp20 phosphorylation and its cardioprotective effects.

\section{Conclusion}

Despite contributing to very low overall cAMP production in heart, loss of AC9 reduces Yotiao- and Hsp20-localized pools of AC activity, but not global PKA phosphorylation. Phosphorylation of Hsp20 occurs largely in ventricles and is vital for the cardioprotective effects of Hsp20 $0^{36-38}$. Deletion of AC9 results in a left ventricular diastolic dysfunction which correlates with reduced Hsp20-associated AC activity and a significant 64\% reduction in baseline Hsp20 phosphorylation. Reductions in AC9 mRNA and protein also occur via microRNA miR-142-3 $\mathrm{p}^{44,45}$. Although normally low in heart, miR-142-3p is upregulated in patients with non-ischemic dilated cardiomyopathy, and in mouse models of hypertrophic cardiomyopathy ${ }^{46-49}$, consistent with a protective role for AC9 in heart.

\section{Materials and Methods}

Generation of AC9 Gene-Targeted Mice. The mouse strain used for this research project, B6;129S5Adcy9Gt(neo)159Lex/Mmucd, identification number 011682-UCD, was obtained from the Mutant Mouse Regional Resource Center, a NIH funded strain repository, and was donated to the MMRRC by Lexicon, Inc. The insertion of the gene trap vector was generated in strain $129 / \mathrm{SvEvBrd-derived} \mathrm{embryonic} \mathrm{stem} \mathrm{cells}{ }^{14}$. The retroviral insertion (5174bp) occurred in the intron between exons 1 and 2 . The chimeric mice were bred to C57BL/6J mice to generate F1 heterozygous animals. Mice were backcrossed with C57BL/6J mice for 7-9 generations. Age matched or wild-type C57BL/6J littermate controls were used as described. All animal protocols were approved by the Institutional Animal Care and Use Committee (IACUC) at the University of Texas Health Science Center at Houston in accordance with the Animal Welfare Act and NIH guidelines.

Genotyping and RT-PCR. Primers used to detect gene trap insertion (WT \#1 and KO \#3; $494 \mathrm{bp}$ ) or WT animals (WT \#1 and \#2; $280 \mathrm{bp}$ ) were as follows: KO \#3, GGCCAAGAACAGATGGAACAG; WT \#1, TCCCTAGCCATTCCTAGCAAAGC; WT \#2, CAGTTCACCTTTTCCATACCCCTAG. Primers used for real-time PCR are from ${ }^{50}$, except AC9. Primer sequences for AC9 are as follows (see Fig. 1): AC9-1 Fwd CGGTCTCCCACAGATGAGAT; AC9-1 Rev, TCTGGGGACAGAAACTGAGG; AC9-2 Fwd, CTTTGATAACCTTAAGACTTGC; AC9-2 Rev, CAGGAGCTGGAGCGATCATA. Real-time PCR was performed and analyzed as described ${ }^{51}$, using GAPDH as a control template.

Plasmids and adenoviruses. Myc-Yotiao-pcDNA3 was previously described ${ }^{3}$. Myc-tagged Hsp20 was purchased from Origene; V5-Hsp20 was a gift from Dr. George Baillie (U of Glasgow). A flag-tag (MDYKDDDDK) plus two residue linker (GA) was inserted in frame at the N-terminus of human AC9 using nested PCR primers. The resulting clone was sequenced and the activity of the tagged protein verified upon expression in HEK293 cells and Sf9 cells. YFP-tagged AC9 was similarly created using flag-AC9 pCDNA3 as the starting construct and replacing the flag-tag with YFP. To create a catalytically inactive AC9, aspartate 399 was mutated to alanine using QuikChange II Site-Directed Mutagenesis Kit (Agilent Technologies). For adenoviral expression, GFP and YFP-tagged catalytically inactive (AC9-D399A, AC9d) were inserted into the Kpn I/XbaI restriction sites of pShuttle-CMV vector. Recombinant adenoviruses were produced according to the manufacturer's instructions (AdEasy Adenoviral Vector Systems, Stratagene). Appropriate clones were selected by RT-PCR and sequenced. Note, although AC9d is expressed as YFP-tagged, YFP is typically cleaved when expressed by adenoviruses in cardiomyocytes and the YFP tag is not detected by WB.

Cell culture and transfections. HEK293 cells were authenticated by ATCC, cultured in Dulbecco's Modified Eagle Medium with 10\% fetal bovine serum, and transfected with the indicated plasmids using Lipofectamine $2000^{3,17}$. Neonatal rat ventricular myocytes (NRVM) were isolated from 1- to 2-day-old Sprague-Dawley rat hearts as previously described ${ }^{52}$. Medium was changed 24 hours after plating and $48-72$ hrs post isolation NRVMs were infected with adenovirus (multiplicity of infection of 50-100) for 50 hrs prior to treatments. Experiments were carried out on at least three separate NRVM isolations. Isoproterenol was stored and diluted in AT buffer ( $100 \mathrm{mM}$ ascorbate and $10 \mathrm{mM}$ thiourea, $\mathrm{pH} 7.4)$.

Western Blotting. Antibodies used for immunoprecipitation and western blotting include rabbit anti-Hsp20 (phospho S16, Abcam), mouse anti-Hsp20 (Hsp20-11) (Santa Cruz Biotechnology), rabbit anti-phospho-Troponin I (Cardiac) (Ser23/24, Cell Signaling), rabbit anti-Troponin I (Cell signaling), 
rabbit anti-phospho-Phospholamban (Ser16, EMD Millipore), mouse anti-Phospholamban Antibody (2D12, ThermoFisher Scientific), rabbit anti-phospho-PKA Substrate (RRXS*/T*) (100G7E) (Cell Signaling), mouse anti- $\beta$-actin (C4, Santa Cruz Biotechnology), rabbit anti- CREB (48H2, Cell Signaling), rabbit anti-phospho-CREB (Ser133, 87G3, Cell signaling), goat anti-cyclase IX (N-18, Santa Cruz Biotechnology), mouse or rabbit anti-AKAP150 (EMD Millipore for western blotting and Santa Cruz Biotechnology for immunoprecipitation) and normal mouse or rabbit IgG (Santa Cruz Biotechnology). Connexin 43 (rabbit) and connexin 45 (mouse) antibodies were a gift from Dr. John O'Brien (UTHealth). The rabbit anti-Yotiao antibody ${ }^{3}$ and mouse anti-AC5 antibodies ${ }^{51}$ were generated and characterized as described. Full-length western blots are shown in Supplemental Fig. S6.

For analysis of PKA phosphorylation in heart, WT and $\mathrm{AC}^{-1-}$ mice were injected with saline or isoproterenol $(2 \mu \mathrm{g} / \mathrm{g}$ body weight, IP $)$. Animals were sacrificed $4 \mathrm{~min}$ later and heart tissue was harvested. Cardiac extracts were prepared in the presence of phosphatase inhibitors. Equal protein supernatants were subjected to western blot analysis as described in figure legends.

Adenylyl Cyclase Activity and IP-AC Assays. Preparation of heart extracts and measurement of AC activity were performed as previously described ${ }^{3,17}$. AC9 activity in WT hearts was estimated from increasing concentrations of the SQ22,536 inhibitor that displays $>100$ fold selectivity for AC5/6 over AC9 ${ }^{16}$. At X concentration of SQ22,536, AC9 activity $=(\mathrm{WT}-\mathrm{KO}) / \%$ AC9 activity remaining at X concentration. Averages of 4 experiments, performed in duplicate or triplicate, using 10,30,100, and 300 $\mu \mathrm{M}$ concentrations of SQ22,536 were used for estimates; a $0.08-2.5 \%$ difference in activity is observed between WT and AC9KO heart membranes. Note, at zero SQ22,536 there is no detectable difference in activity. Immunoprecipitation of AKAP or Hsp20 complexes followed by western blotting or measurement of associated AC activity was performed as described ${ }^{53}$. AC activity was stimulated with the indicated reagents and cAMP was detected by enzyme immunoassay (Assay Designs) or using $\left[\gamma^{32} \mathrm{P}\right] \mathrm{ATP}$

Proximity Ligation Assay. In situ PLA was performed using a Duolink kit (Sigma-Aldrich, cat. DUO92101) following the manufacturer's protocol. HEK293 cells were cultured on clear bottom 96 well plates (Greiner Bio-One), transfected with the required plasmids and fixed with 4\% PFA. After washing the plate 3 times with PBS, the cells were blocked ( $1 \%$ BSA $+0.075 \%$ Triton X100) for $1 \mathrm{~h}$ at room temperature and then incubated with primary antibodies overnight. Antibodies included: mouse anti-Hsp20 (SC-51955, SantaCruz, 1:500), mouse

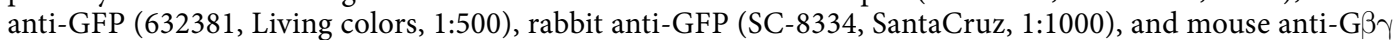
(SC-378, SantaCruz, 1:1000). After removal of primary antibodies, the samples were incubated with anti-mouse PLUS and anti-rabbit MINUS PLA probes for $1 \mathrm{~h}$ at $37^{\circ} \mathrm{C}$. Subsequent steps of ligation and amplification were according to the manufacturer's protocol. After the last wash, cells were stained with DAPI ( $1 \mu \mathrm{g} / \mathrm{ml})$ and imaged using an epifluorescence high content imaging microscope with a 20X objective (CellInsight CX5 High Content Screening platform, ThermoFisher). Data analysis was performed using FACS analysis software (FlowJo, USA). To prevent false positives, cells with saturating YFP fluorescence or less than 4 positive signals (dots) per cell were not considered in the analysis.

Echocardiography. AC9 knockout and WT mice (3-7 months) were anesthetized in a chamber under $2.5 \%$ isoflurane. After sedation, mice were transferred to a heated platform and fixed in the prone position for electrocardiogram recordings and cardiac imaging. Anesthesia was administered via a nose cone at $1-1.5 \%$ isoflurane during recordings. Cardiac imaging was utilized to assess left ventricular (LV) diastolic function and other key cardiac parameters. Initial analysis of LV diastolic function was evaluated with pulsed-wave Doppler (PWD) (20 MHz, Doppler Signaling Processing Workstation (DSPW), Indus Instruments, Webster, USA) of the mitral valve (MV) inflow ${ }^{54}$. Key parameters of the mitral valve flow profile including, early (E) and late (A) filling velocities, aortic ejection time (AET), isovolumetric relaxation time (IVRT), and isovolumetric contraction time (IVCT) were determined from recordings with DSPW. The ratio of the E/A velocities was used to assess changes in LV filling.

Further evaluation of LV diastolic function and key cardiac function used high resolution ultrasound (40 MHz; Vevo 3100, Visual Sonics Inc., Toronto, Canada); analysis was performed in VevoLab (VisualSonic). $\mathrm{LV}$ volume and mass, ejection fraction (EF), and fractional shortening (FS) were calculated from recordings of the short axis using M-mode imaging. Additional measurements from M-mode imaging included the thickness of the LV anterior and posterior wall (LVAW and LVPW) during both systole and diastole. Diastolic function was assessed with both PWD and tissue Doppler imaging (TDI), visualized in apical four-chamber view. IVCT, IVRT, AET, E and A velocities, myocardial performance index (MPI), and E/A ratios were determined from PWD recordings. TDI from the septal side of the mitral annulus were used to assess the early (E') and late (A') velocities of the mitral annulus and the ratio of E'/A', A'/E', and MV E/E'. All measurements were averaged from 5 cardiac cycles for each parameter. Note, differences in MV E/A and E'/A' ratios are likely underestimates since the A' and MV A measurements were not always possible on WT mice with faster heart rates where the early wave is dominant; this was never an issue with $\mathrm{AC} 9^{-1-}$ mice.

Statistical Analysis. Data are expressed as mean \pm standard error of the mean (SEM), except where noted. Differences between samples were determined using two-way analysis of variance (ANOVA) followed by Bonferroni's Multiple Comparison Test for comparison between multiple groups, or unpaired t test for comparison between two groups. Chi square test was used to compare observed and expected genotype frequencies. Significant $\mathrm{p}$ values are indicated as follows: $(*)$ denotes a $\mathrm{p}$ value $<0.05,(* *)<0.01$ and $(* * *)<0.001$. All analyses were performed using Excel or SigmaPlot statistical analysis software. 


\section{References}

1. Sadana, R. \& Dessauer, C. W. Physiological Roles for G Protein-Regulated Adenylyl Cyclase Isoforms: Insights from Knockout and Overexpression Studies. NeuroSignals 17, 5-22 (2009).

2. Timofeyev, V. et al. Adenylyl cyclase subtype-specific compartmentalization: differential regulation of L-type Ca2+ current in ventricular myocytes. Circ Res 112, 1567-1576 (2013).

3. Piggott, L. A., Bauman, A. L., Scott, J. D. \& Dessauer, C. W. The A-kinase anchoring protein Yotiao binds and regulates adenylyl cyclase in brain. Proc Natl Acad Sci USA 105, 13835-13840 (2008).

4. Li, Y., Chen, L., Kass, R. S. \& Dessauer, C. W. The A-kinase anchoring protein Yotiao facilitates complex formation between type 9 adenylyl cyclase and the IKs potassium channel in heart. J Biol Chem 287, 29815-29824 (2012).

5. Efendiev, R. \& Dessauer, C. W. A kinase-anchoring proteins and adenylyl cyclase in cardiovascular physiology and pathology. $J$ Cardiovasc Pharmacol 58, 339-344 (2011).

6. Scott, J. D., Dessauer, C. W. \& Tasken, K. Creating order from chaos: cellular regulation by kinase anchoring. Annu Rev Pharmacol Toxicol 53, 187-210 (2013).

7. Dessauer, C. W. Adenylyl cyclase-A-kinase anchoring protein complexes: the next dimension in cAMP signaling. Mol Pharmacol 76, 935-941 (2009).

8. Efendiev, R., Bavencoffe, A., Hu, H., Zhu, M. X. \& Dessauer, C. W. Scaffolding by A-kinase anchoring protein enhances functional coupling between adenylyl cyclase and TRPV1 channel. J Biol Chem 288, 3929-3937 (2013).

9. Ackerman, M. J. \& Mohler, P. J. Defining a new paradigm for human arrhythmia syndromes: phenotypic manifestations of gene mutations in ion channel- and transporter-associated proteins. Circ Res 107, 457-465 (2010).

10. Qian, J. et al. Blockade of Hsp20 phosphorylation exacerbates cardiac ischemia/reperfusion injury by suppressed autophagy and increased cell death. Circ Res 105, 1223-1231 (2009).

11. Edwards, H. V., Scott, J. D. \& Baillie, G. S. PKA phosphorylation of the small heat-shock protein Hsp20 enhances its cardioprotective effects. Biochem Soc Trans 40, 210-214 (2012).

12. Nicolaou, P. et al. Human mutation in the anti-apoptotic heat shock protein 20 abrogates its cardioprotective effects. J Biol Chem $\mathbf{2 8 3}$, 33465-33471 (2008).

13. Antoni, F. A. Adenylyl cyclase type 9. UCSD-Nature Molecule Pages. doi:10.1038/mp.a000131.000101 (2006).

14. International Mouse Knockout, C., Collins, F. S., Rossant, J. \& Wurst, W. A mouse for all reasons. Cell 128, 9-13 (2007).

15. Ostrom, R. S. et al. Angiotensin II enhances adenylyl cyclase signaling via Ca2+/calmodulin. Gq-Gs cross-talk regulates collagen production in cardiac fibroblasts. J Biol Chem 278, 24461-24468 (2003).

16. Brand, C. S., Hocker, H. J., Gorfe, A. A., Cavasotto, C. N. \& Dessauer, C. W. Isoform selectivity of adenylyl cyclase inhibitors: characterization of known and novel compounds. J Pharmacol Exp Ther 347, 265-275 (2013).

17. Efendiev, R. et al. AKAP79 interacts with multiple adenylyl cyclase (AC) isoforms and scaffolds AC5 and -6 to alpha-amino-3hydroxyl-5-methyl-4-isoxazole-propionate (AMPA) receptors. J Biol Chem 285, 14450-14458 (2010).

18. Severs, N. J. et al. Gap junction alterations in human cardiac disease. Cardiovasc Res 62, 368-377 (2004).

19. Fan, G. C., Chu, G. \& Kranias, E. G. Hsp20 and its cardioprotection. Trends Cardiovasc Med 15, 138-141 (2005).

20. Beall, A. et al. The small heat shock-related protein, HSP20, is phosphorylated on serine 16 during cyclic nucleotide-dependent relaxation. J Biol Chem 274, 11344-11351 (1999).

21. Brand, C. S., Sadana, R., Malik, S., Smrcka, A. V. \& Dessauer, C. W. Adenylyl Cyclase 5 Regulation by Gbetagamma Involves IsoformSpecific Use of Multiple Interaction Sites. Mol Pharmacol 88, 758-767 (2015).

22. Paterson, J. M., Smith, S. M., Harmar, A. J. \& Antoni, F. A. Control of a novel adenylyl cyclase by calcineurin. Biochem.Biophys.Res. Commun. 214, 1000 (1995).

23. Dessauer, C. W. et al. International Union of Basic and Clinical Pharmacology. CI. Structures and Small Molecule Modulators of Mammalian Adenylyl Cyclases. Pharmacol Rev 69, 93-139 (2017).

24. Antoni, F. A. et al. Ca2+/calcineurin-inhibited adenylyl cyclase, highly abundant in forebrain regions, is important for learning and memory. Journal of Neuroscience 18, 9650-9661 (1998).

25. Liu, L., Das, S., Losert, W. \& Parent, C. A. mTORC2 regulates neutrophil chemotaxis in a cAMP- and RhoA-dependent fashion. Dev Cell 19, 845-857 (2010).

26. Small, K. M. et al. An Ile to Met polymorphism in the catalytic domain of adenylyl cyclase type 9 confers reduced beta2-adrenergic receptor stimulation. Pharmacogenetics 13, 535-541 (2003).

27. Toyota, T. et al. Molecular analysis, mutation screening, and association study of adenylate cyclase type 9ene (ADCY9) in mood disorders. Am J Med Genet 114, 84-92 (2002).

28. Berndt, S. I. et al. Genome-wide meta-analysis identifies 11 new loci for anthropometric traits and provides insights into genetic architecture. Nature genetics 45, 501-512 (2013).

29. Nichols, C. B. et al. Sympathetic stimulation of adult cardiomyocytes requires association of AKAP5 with a subpopulation of L-type calcium channels. Circ Res 107, 747-756 (2010).

30. Swan, H. et al. Sinus node function and ventricular repolarization during exercise stress test in long QT syndrome patients with KvLQT1 and HERG potassium channel defects. J Am Coll Cardiol 34, 823-829 (1999).

31. Schwartz, P. J. et al. Neural control of heart rate is an arrhythmia risk modifier in long QT syndrome. J Am Coll Cardiol 51, 920-929 (2008).

32. Honore, E. et al. Cloning, expression, pharmacology and regulation of a delayed rectifier $\mathrm{K}+$ channel in mouse heart. EMBO J 10, 2805-2811 (1991).

33. Salama, G., Baker, L., Wolk, R., Barhanin, J. \& London, B. Arrhythmia phenotype in mouse models of human long QT. J Interv Card Electrophysiol 24, 77-87 (2009).

34. Marx, S. O. et al. Requirement of a macromolecular signaling complex for beta adrenergic receptor modulation of the KCNQ1KCNE1 potassium channel. Science 295, 496-499 (2002).

35. Tosaka, T. et al. Nicotine induces a long QT phenotype in Kcnq1-deficient mouse hearts. J Pharmacol Exp Ther 306, $980-987$ (2003).

36. Fan, G. C. \& Kranias, E. G. Small heat shock protein 20 (HspB6) in cardiac hypertrophy and failure. J Mol Cell Cardiol 51, 574-577 (2011).

37. Martin, T. P., Currie, S. \& Baillie, G. S. The cardioprotective role of small heat-shock protein 20. Biochem Soc Trans 42, 270-273 (2014).

38. Fan, G. C. et al. Heat shock protein 20 interacting with phosphorylated Akt reduces doxorubicin-triggered oxidative stress and cardiotoxicity. Circ Res 103, 1270-1279 (2008).

39. Martin, T. P. et al. Targeted disruption of the heat shock protein 20-phosphodiesterase 4D (PDE4D) interaction protects against pathological cardiac remodelling in a mouse model of hypertrophy. FEBS Open Bio 4, 923-927 (2014).

40. Dreiza, C. M. et al. The small heat shock protein, HSPB6, in muscle function and disease. Cell Stress Chaperones 15, 1-11 (2010).

41. Sin, Y. Y., Martin, T. P., Wills, L., Currie, S. \& Baillie, G. S. Small heat shock protein 20 (Hsp20) facilitates nuclear import of protein kinase D 1 (PKD1) during cardiac hypertrophy. Cell Commun Signal 13, 16 (2015).

42. Edwards, H. V., Scott, J. D. \& Baillie, G. S. The A-kinase-anchoring protein AKAP-Lbc facilitates cardioprotective PKA phosphorylation of Hsp20 on Ser(16). Biochem J 446, 437-443 (2012).

43. Leren, I. S. et al. Cardiac Mechanical Alterations and Genotype Specific Differences in Subjects With Long QT Syndrome. JACC: Cardiovascular Imaging 8, 501-510 (2015). 
44. Huang, B. et al. miR-142-3p restricts cAMP production in CD4+ CD25- T cells and CD4+ CD25+ TREG cells by targeting AC9 mRNA. EMBO Rep 10, 180-185 (2009).

45. Lv, M. et al. An oncogenic role of miR-142-3p in human T-cell acute lymphoblastic leukemia (T-ALL) by targeting glucocorticoid receptor-alpha and cAMP/PKA pathways. Leukemia 26, 769-777 (2012).

46. Tijsen, A. J., Pinto, Y. M. \& Creemers, E. E. Circulating microRNAs as diagnostic biomarkers for cardiovascular diseases. Am J Physiol Heart Circ Physiol 303(9), H1085-1095 (2012).

47. Voellenkle, C. et al. MicroRNA signatures in peripheral blood mononuclear cells of chronic heart failure patients. Physiol Genomics 42, 420-426 (2010).

48. Bagnall, R. D., Tsoutsman, T., Shephard, R. E., Ritchie, W. \& Semsarian, C. Global MicroRNA Profiling of the Mouse Ventricles during Development of Severe Hypertrophic Cardiomyopathy and Heart Failure. PLoS One 7, e44744 (2012).

49. Baskerville, S. \& Bartel, D. P. Microarray profiling of microRNAs reveals frequent coexpression with neighboring miRNAs and host genes. RNA 11, 241-247 (2005).

50. Landa, L. R. Jr. et al. Interplay of Ca2+ and cAMP signaling in the insulin-secreting MIN6 beta-cell line. J Biol Chem 280, 31294-31302 (2005).

51. Bavencoffe, A. et al. Persistent Electrical Activity in Primary Nociceptors after Spinal Cord Injury Is Maintained by Scaffolded Adenylyl Cyclase and Protein Kinase A and Is Associated with Altered Adenylyl Cyclase Regulation. J Neurosci 36, 1660-1668 (2016).

52. Wu, H. C. et al. Identification and characterization of two ankyrin-B isoforms in mammalian heart. Cardiovascular Research 107, 466-477 (2015).

53. Li, Y. \& Dessauer, C. W. In Cyclic Nucleotide Signaling (eds Cheng, X.) Ch. 9, 147-164 (CRC Press, 2015).

54. Reddy, A. K. et al. Pulsed Doppler signal processing for use in mice: design and evaluation. IEEE Trans Biomed Eng 52, 1764-1770 (2005).

\section{Acknowledgements}

The authors thank John O'Brien for connexin antibodies, the Mouse Cardiovascular Phenotyping Core Laboratory at UTHealth, and Andrew Peters, Giovanni Davogustto, and Heinrich Taegtmeyer for their assistance with cardiac imaging and many helpful discussions. This work was supported by National Institutes of Health Grants RO1GM60419 (CWD) and T32 GM089657 (TAB) and American Heart Association 16GRNT30410011 (SRC).

\section{Author Contributions}

Y.L., T.A.B., C.S.B., A.G.C., S.R.C. and C.W.D. designed research; Y.L., T.A.B., Y.W., J.S., A.G.C., S.R.C. and C.S.B. performed research; Y.L., T.A.B., Y.W., C.S.B., A.G.C. and C.W.D. analyzed data; and C.W.D. wrote the paper.

\section{Additional Information}

Supplementary information accompanies this paper at doi:10.1038/s41598-017-05816-w

Competing Interests: The authors declare that they have no competing interests.

Publisher's note: Springer Nature remains neutral with regard to jurisdictional claims in published maps and institutional affiliations.

(c) (i) Open Access This article is licensed under a Creative Commons Attribution 4.0 International

License, which permits use, sharing, adaptation, distribution and reproduction in any medium or format, as long as you give appropriate credit to the original author(s) and the source, provide a link to the Creative Commons license, and indicate if changes were made. The images or other third party material in this article are included in the article's Creative Commons license, unless indicated otherwise in a credit line to the material. If material is not included in the article's Creative Commons license and your intended use is not permitted by statutory regulation or exceeds the permitted use, you will need to obtain permission directly from the copyright holder. To view a copy of this license, visit http://creativecommons.org/licenses/by/4.0/.

(c) The Author(s) 2017 